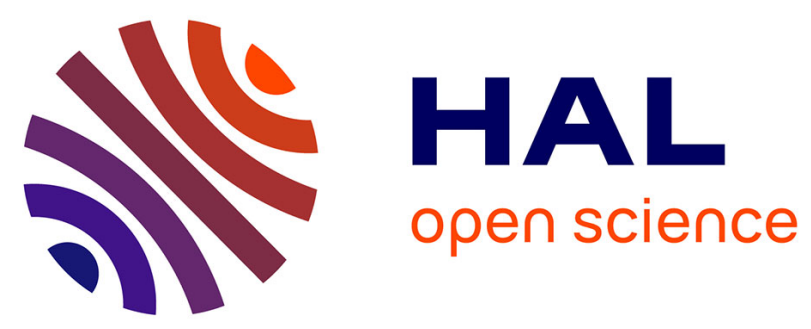

\title{
Asymptotic Analysis of Congested Communication Networks
}

\author{
J. Frederic Bonnans, Mounir Haddou
}

\section{To cite this version:}

J. Frederic Bonnans, Mounir Haddou. Asymptotic Analysis of Congested Communication Networks. [Research Report] RR-3133, INRIA. 1997. inria-00073556

\section{HAL Id: inria-00073556 \\ https://hal.inria.fr/inria-00073556}

Submitted on 24 May 2006

HAL is a multi-disciplinary open access archive for the deposit and dissemination of scientific research documents, whether they are published or not. The documents may come from teaching and research institutions in France or abroad, or from public or private research centers.
L'archive ouverte pluridisciplinaire HAL, est destinée au dépôt et à la diffusion de documents scientifiques de niveau recherche, publiés ou non, émanant des établissements d'enseignement et de recherche français ou étrangers, des laboratoires publics ou privés. 
INSTITUT NATIONAL DE RECHERCHE EN INFORMATIQUE ET EN AUTOMATIQUE

\section{Asymptotic analysis of congested communication networks}

J. Frédéric Bonnans, Mounir Haddou

$\mathbf{N}^{\circ} 3133$

Mars 1997

THÈME 4 



\title{
RIN RIA
}

\section{Asymptotic analysis of congested communication networks}

\author{
J. Frédéric Bonnans*, Mounir Haddou ${ }^{\dagger}$ \\ Thème 4 - Simulation et optimisation \\ de systèmes complexes \\ Projet PROMATH \\ Rapport de recherche $\mathrm{n}^{\circ} 3133$ - Mars 1997 - 19 pages
}

\begin{abstract}
This paper is devoted to the study of a routing problem in telecommunication networks, when the cost function is the average delay. We establish asymptotic expansions for the value function and solutions in the vicinity of a congested nominal problem. The study is strongly related to the one of a partial inverse barrier method for linear programming.
\end{abstract}

Key-words: Telecommunication networks, multicommodity flows, asymptotic expansions, linear programming, perturbation analysis, barrier functions, penalty methods.

(Résumé : tsvp)

* INRIA, B.P. 105, 78153 Rocquencourt, France. Email: Frederic.Bonnans@inria.fr.

$\dagger$ INRIA, B.P. 105, 78153 Rocquencourt, France. Email: Mounir.Haddou@inria.fr. 


\section{Analyse asymptotique des réseaux de communications congestionnés}

Résumé : Nous étudions le problème de minimisation du délai de routage dans les réseaux de télécommunications. Nous établissons des développements asymptotiques pour la fonction coût et les solutions pour des réseaux congestionnés. Il s'agit d'un problème d'analyse de perturbations singulières et où chaque problème perturbé peut avoir plusieurs solutions. Cette étude est très proche de celle de la méthode de barrière inverse partielle en programmation linéaire.

Mots-clé : Réseaux de télécommunications, multiflots, développements asymptotiques, programmation linéaire, analyse de perturbations, fonctions barrières, méthodes de pénalités. 
AMS subject classifications 49M37, 65K05, 65Y05, 90B12, 90C05, 90C25

\section{Introduction}

This paper is devoted to the study of the problem of minimizing the average delay in low-rate packet-switched networks. This problem is described in detail in the next section. For the moment it suffices to say that the equations are those of a multicommodity flows problem, while the cost function is the sum over of the inverse of the residual capacities on each arc, weighted by the capacity of that arc. This is a convex problem that may have several solutions.

We consider the problem of computing the expansion of the value function and solution of this problem, taking as perturbation variables the arc capacities. We assume that the unperturbed problem is such that some arcs are congested, i.e. their residual capacities are null, and we discuss the effect of a variation of capacities that is linear with respect to a scalar parameter $t, t=0$ corresponding to the unperturbed problem. We assume that congestion does not occur for positive $t$.

This is a particular case of the general problem of computing the expansion of value function and solution of a nonlinear programming problem. This is a very active field, see e.g. the review [4] and the book [6]. In particular, there exist formulas, based on first-order information, for the marginal value of a convex problem under fairly weak hypotheses. For computing the second-order expansion of the cost and the first-order expansion of solutions, it is necessary to use second-order information. However, most the theory of expansions of solutions deals with the case of (locally) unique solutions, and the perturbation theory, as well as the theory of second-order optimality conditions, is still in infancy [3, 12].

In addition, the main difficulty comes from the fact that the unperturbed problem is singular (due to the congestion). Therefore we cannot use much the results of the literature, rather we had to tailor specific estimates.

The results of this paper are obtained by studying an auxiliary problem $\left(P_{\varepsilon}\right)$, with $\varepsilon>0$, in which the perturbation parameter $t$ is considered as an optimization variable, and penalized with weight $\varepsilon^{-1}$. The advantage of this transformation is to ease comparisons with the literature devoted to the central path, i.e. the set of points that minimize a given barrier function. The central path is one of the basic concepts of the theory of interior point algorithms $[8,11]$. A local analysis of central paths associated with general penalty functions may be found in [2]. The perturbation problem studied here is similar (although not identical) to a partial inverse barrier method (P.I.B) in linear programming. That is, a method in which a penalty term, proportional to the inverse of slack variables, is applied to some inequalities, but not all. The inverse barrier method was introduced by Carroll [5] and is studied in [7]. As possible motivation of partial barrier methods is as follows. For large scale optimization problem with a small number of coupling variables (i.e. a recourse problem) it might be of interest to apply a penalty barrier to the constraints on the coupling variables when only a small number of constraints of the decoupled problems is active (and therefore it is not efficient to deal explicitely with all of them).

$\mathrm{RR} \mathrm{n}^{\circ} 3133$ 
The paper is organized as follows. The next section is devoted to the presentation of the problem. In $\S 3$ we obtain some preliminary estimates for some special situations like problems in the motivation subsection. In the next section we prove some convergence results. Asymptotic expansions of the solutions and the cost function are given in Section 4 and 5 . The last section discusses some extensions.

\section{Minimizing the average delay in low-rate packet -switched networks}

We now describe the problem of minimizing the average delay in low-rate packet-switched networks [10] when small changes can be made in capacities.

Such a network can be modeled as an oriented graph $G=(N, L)$, where $N$ and $L$ are the nodes and arcs sets, with capacities $\gamma_{i}$ on each arc $l_{i}$. We denote $|N|=n$ and $|L|=p$, and associate with a demand $d^{k}$ of value $v^{k}$, between a source and sink nodes $o^{k}$ and $s^{k}$, a commodity $k$ represented by a vector $x^{k} \in \mathbb{R}^{p}$. Each component $x_{i}^{k}$ is the amount of commodity $k$ which flows through the link $l_{i}$. The problem is then

$$
\begin{array}{r}
\operatorname{Min}_{x} \sum_{1 \leq i \leq p} \frac{\gamma_{i}}{x_{i}^{0}}, \quad \gamma_{i}-\sum_{1 \leq k \leq K} x_{i}^{k}=x_{i}^{0}, i=1, \ldots, p, \\
A x^{k}=b^{k}, \quad k=1, \ldots, K, \quad x^{k} \geq 0, k=0, \ldots, K .
\end{array}
$$

Here $A$ is the incidence matrix of the graph $G$ and $b^{k} \in \mathbb{R}^{n}$ is such that

$$
b_{i}^{k}=-v^{k} \text { if } i=o^{k}, \quad b_{i}^{k}=v^{k} \text { if } i=s^{k}, \quad b_{i}^{k}=0 \text { otherwise. }
$$

The cost function represents the sum of global delays occurring for packets sent through the network.

Let us now consider the influence of a variation of arc capacities on the solution. In order to obtain an expansion of the solution, we assume that the capacity on arc $i$ is now augmented of an amount $t \omega_{i}$, where $t \geq 0$ is a scalar number. The considered problems are

$$
\begin{gathered}
\operatorname{Min}_{x} \sum_{1 \leq i \leq p} \frac{\gamma_{i}+t \omega_{i}}{x_{i}^{0}}, \quad \gamma_{i}+t \omega_{i}-\sum_{1 \leq k \leq K} x_{i}^{k}=x_{i}^{0}, i=1, \ldots, p, \\
A x^{k}=b^{k}, \quad k=1, \ldots, K, \quad x^{k} \geq 0, k=0, \ldots, K,
\end{gathered}
$$

for any value (relatively small) of the additional variable $t$.

We find convenient to introduce an artificial variable $\varepsilon$ and consider the family of problems

$$
\begin{aligned}
& \operatorname{Min}_{x, t} t+\varepsilon \sum_{1 \leq i \leq p} \frac{\gamma_{i}+t \omega_{i}}{x_{i}^{0}}, \quad \gamma_{i}+t \omega_{i}-\sum_{1 \leq k \leq K} x_{i}^{k}=x_{i}^{0}, i=1, \ldots, p, \\
& A x^{k}=b^{k}, k=1, \ldots, K, \quad t \geq 0, x^{k} \geq 0, k=0, \ldots, K .
\end{aligned}
$$

We will denote the cost function of the above problem as $f_{\varepsilon}(x, t):=t+\varepsilon \sum_{1 \leq i \leq p} \frac{\gamma_{i}+t \omega_{i}}{x_{i}^{0}}$. 
This problem may be interpreted as a penalization of the perturbation parameter $t$. Observe that this problem is very close to an inverse barrier method applied to the linear program obtained by taking $\varepsilon=0$, the barrier being applied only to the coupling constraints.

Notations Let $x \in \mathbb{R}^{n}$. The relation $x>0$ means $x_{i}>0, i=1,2, \cdots, n$, while $x \geq 0$ means $x_{i} \geq 0, i=1,2, \cdots, n$. We denote $x^{+}$the nonnegative part of $x$, i.e. $x_{i}^{+}:=$ $\max \left(x_{i}, 0\right), i=1,2, \cdots, n$. Let $J$ be a subset of $I:=\{1, \cdots, p\}$. We denote $\bar{J}:=I \backslash J$ and $x_{J}:=\left\{x_{i}, i \in J\right\}$. By $\|$.$\| we mean the Euclidean norm \|\cdot\|_{2}$ in the space given by the context.

The notation $x(\varepsilon)=O(\varepsilon)$ (resp. $x(\varepsilon)=o(\varepsilon)$ ) means that there is a constant $K$ (dependent on problem data) such that for every $\varepsilon>0,\|x(\varepsilon)\| \leq K \varepsilon$ (resp. $\lim _{\varepsilon \downarrow 0} \varepsilon^{-1}\|x(\varepsilon)\|=$ $0)$. We will also denote $x(\varepsilon) \approx \varepsilon$ when $x(\varepsilon)=O(\varepsilon)$ and $\|x(\varepsilon)\|^{-1}=O(\varepsilon)$. Let $(P)$ be an optimization problem. By $F(P), S(P)$ and $v(P)$ we denote respectively the feasible set, the optimal set and the optimal value.

\section{Preliminary estimates}

Consider the linear programming problem obtained by setting $\varepsilon=0$ in $\left(P_{\varepsilon}\right)$ :

$$
\begin{array}{lr}
\operatorname{Min}_{x, t} t, & \gamma_{i}+t \omega_{i}-\sum_{1 \leq k \leq K} x_{i}^{k}=x_{i}^{0}, i=1, \ldots, p, \\
A x^{k}=b^{k}, \quad k=1, \ldots, K, \quad t \geq 0, \quad x^{k} \geq 0, k=0, \ldots, K .
\end{array}
$$

We assume throughout the paper that the following assumptions are satisfied:

(i) the optimal value $v(L P)=0$, and

(ii) the following congestion condition is satisfied:

$$
\exists j \in I \text { such that } \forall(x, 0) \in S(L P), \quad x_{j}^{0}=0 .
$$

With $(x, t) \in F(L P)$ we associate the sets of active constraints

$$
I^{k}(x, t):=\left\{i \in I: x_{i}^{k}=0\right\}, \quad k=0, \ldots, K .
$$

The sets of constraints that are active for all solutions of $(L P)$ are denoted

$$
I_{0}^{k}:=\bigcap_{(x, 0) \in S(L P)} I^{k}(x, 0), \quad k=0, \ldots, K .
$$

These sets determine the solution set $S(L P)$ and its relative interior by

$$
S(L P)=\left\{(x, 0) \in F(L P): I_{0}^{k} \subset I^{k}(x, 0), \quad k=0, \ldots, K\right\},
$$




$$
\operatorname{ri} S(L P)=\left\{(x, 0) \in F(L P): I^{k}(x, 0)=I_{0}^{k}, \quad k=0, \ldots, K\right\} .
$$

The family of problems $\left(P_{\varepsilon}\right)$ can be interpreted as a partial inverse barrier (P.I.B) method for solving (LP). Indeed, the second part of the cost function $f_{\varepsilon}$ implicitely implies that $x^{0}$ must be nonnegative. We can then consider it as a barrier applied to the components $x^{0}$. The results of this section are very closed to those concerning the inverse barrier method introduced by Carroll [5] and studied in [7]. Convergence of this well known method and some other penalty and barrier methods to a particular optimal solution is also proved in [2].

We define the set of partial centers of $S(L P)$ as the optimal set of

$$
\underset{(x, 0) \in S(L P)}{\operatorname{Min}} \sum_{i \notin I_{0}^{0}} \frac{\gamma_{i}}{x_{i}^{0}} .
$$

Using the fact that $S(L P)$ is nonempty and bounded, it may be checked by standard arguments that $S(R)$ is nonempty, bounded, closed and convex. Since $F(R)=S(L P)$, every partial center $(x, 0)$ satisfies

$$
x_{i}^{k}=0 \quad \text { if } \quad i \in I_{0}^{k}, \quad k=0, \ldots, K .
$$

Furthermore, as the cost function of $(R)$ is strictly convex with respect to components $x_{i}^{0}$ for $i \notin I_{0}^{0}$, all partial centers have the same components $x_{i}^{0}$ for $i \notin I_{0}^{0}$.

To begin our discussion, we recall the celebrated Hoffmann's lemma [9].

Lemma 3.1 Let $C:=\left\{z \in \mathbb{R}^{n}: E z=e, G z \leq g\right\}$ be a nonempty set. There exists a constant $\delta>0$ depending only on the matrices $E$ and $G$ such that

$$
\forall z \in \mathbb{R}^{n}, \quad \operatorname{dist}(z, C) \leq \delta\left(\left\|(G z-g)^{+}\right\|+\|E z-e\|\right) .
$$

By $(x(\varepsilon), t(\varepsilon))$ we denote an arbitrary solution of $\left(P_{\varepsilon}\right)$. The next lemma gives the order of magnitude of $t(\varepsilon)$.

Lemma 3.2 One has $t(\varepsilon) \approx \sqrt{\varepsilon}$.

Proof. Let $(x, 0) \in S(L P)$ and define $\left(y_{\varepsilon}, t_{\varepsilon}\right)$ by

$$
y_{\varepsilon}^{k}=x^{k} \quad \text { for } k=1, \ldots, K, \quad t_{\varepsilon}=\sqrt{\varepsilon} \quad \text { and } \quad y_{\varepsilon}^{0}=x^{0}+\sqrt{\varepsilon} \omega .
$$

Obviously $\left(y_{\varepsilon}, t_{\varepsilon}\right) \in F\left(P_{\varepsilon}\right)$. Therefore

$$
t(\varepsilon) \leq v\left(P_{\varepsilon}\right) \leq f_{\varepsilon}\left(y_{\varepsilon}, t_{\varepsilon}\right)=O(\sqrt{\varepsilon}) .
$$

On the other hand, applying Lemma 3.1 to $C=S(L P)$ and $z=(x(\varepsilon), t(\varepsilon))$, we obtain that

$$
\exists \delta_{1}>0 \text { such that } \operatorname{dist}((x(\varepsilon), t(\varepsilon)), S(L P))=\delta_{1} t(\varepsilon) .
$$


In particular, for the components $x_{I_{0}^{0}}^{0}(\varepsilon)$, we can conclude that

$$
\left|x_{i}^{0}(\varepsilon)\right| \leq \delta_{1} t(\varepsilon), \quad i \in I_{0}^{0} .
$$

It follows that

$$
O(\sqrt{\varepsilon}) \geq v\left(P_{\varepsilon}\right)=f_{\varepsilon}(x(\varepsilon), t(\varepsilon)) \geq \varepsilon \sum_{i \in I_{0}^{0}} \frac{\gamma_{i}}{x_{i}^{0}(\varepsilon)} \geq \frac{\left|I_{0}^{0}\right|}{\delta_{1}} \frac{\varepsilon}{t(\varepsilon)}
$$

and then $t(\varepsilon) \approx \sqrt{\varepsilon}$. $\quad$

Lemma 3.3 The solution sets $\left\{S\left(P_{\varepsilon}\right)\right\}_{\varepsilon>0}$ are uniformely bounded for $\varepsilon$ close to 0 , and any limit-point $(\bar{x}, \bar{t})$ of $\{(x(\varepsilon), t(\varepsilon))\}_{\varepsilon>0}$ with $(x(\varepsilon), t(\varepsilon)) \in S\left(P_{\varepsilon}\right)$, is a partial center.

Proof. By Lemma $3.2, t(\varepsilon) \approx \sqrt{\varepsilon}$. In particular, for $\varepsilon$ small enough, $t(\varepsilon) \leq 1$ and

$$
S\left(P_{\varepsilon}\right) \subset Z:=\left\{(x, t): 0 \leq t \leq 1 \quad \text { and } \quad 0 \leq x^{k} \leq \gamma+\omega \quad k=0, \ldots, K\right\} .
$$

As $Z$ is bounded, uniform boundedness of $S\left(P_{\varepsilon}\right)$ follows.

Let $(\bar{x}, \bar{t})$ be a limit-point of $\{(x(\varepsilon), t(\varepsilon))\}_{\varepsilon>0}$. As $F(L P)$ is closed and $t(\varepsilon) \approx \sqrt{\varepsilon}, \bar{t}=0$ and $(\bar{x}, \bar{t})=(\bar{x}, 0) \in S(L P)$.

Now let $\varepsilon_{k} \downarrow 0$ be such that there exists $\left\{\left(x\left(\varepsilon_{k}\right), t\left(\varepsilon_{k}\right)\right)\right\} \in S\left(P_{\varepsilon_{k}}\right)$ converging to $(\bar{x}, 0)$. Let $(\hat{x}, 0) \in \operatorname{ri} S(L P)$. Then

$$
(\hat{x}, 0)=\lim _{k \rightarrow+\infty}\left(\hat{x}\left(\varepsilon_{k}\right), t\left(\varepsilon_{k}\right)\right) \quad \text { with } \quad \hat{x}\left(\varepsilon_{k}\right):=x\left(\varepsilon_{k}\right)+\hat{x}-\bar{x} .
$$

As $(\hat{x}, 0) \in \operatorname{ri} S(L P)$, we have $x_{i}^{0}\left(\varepsilon_{k}\right)=\hat{x}_{i}^{0}\left(\varepsilon_{k}\right)$ whenever $i \in I_{0}^{0}$ and $\left(\hat{x}\left(\varepsilon_{k}\right), \hat{t}\left(\varepsilon_{k}\right)\right) \in F(L P)$ for $\varepsilon_{k}$ small enough.

From $f_{\varepsilon_{k}}\left(x\left(\varepsilon_{k}\right), t\left(\varepsilon_{k}\right)\right) \leq f_{\varepsilon_{k}}\left(\hat{x}\left(\varepsilon_{k}\right), t\left(\varepsilon_{k}\right)\right)$, we deduce that

$$
\sum_{i \notin I_{0}^{0}} \frac{\gamma_{i}+t\left(\varepsilon_{k}\right) \omega_{i}}{x_{i}^{0}\left(\varepsilon_{k}\right)} \leq \sum_{i \notin I_{0}^{0}} \frac{\gamma_{i}+t\left(\varepsilon_{k}\right) \omega_{i}}{\hat{x}_{i}^{0}\left(\varepsilon_{k}\right)} .
$$

Passing to the limit in (3.4), we obtain that $\bar{x}_{i}^{0}>0$ whenever $i \notin I_{0}^{0}$ and

$$
\sum_{i \notin I_{0}^{0}} \frac{\gamma_{i}}{\bar{x}_{i}^{0}} \leq \sum_{i \notin I_{0}^{0}} \frac{\gamma_{i}}{\hat{x}_{i}^{0}} .
$$

This proves that $(\bar{x}, 0)$ is a partial center. $\square$

$\mathrm{RR} \mathrm{n}^{\circ} 3133$ 


\section{Asymptotic expansion of the solutions}

Let $\left(x^{*}, 0\right)$ be a fixed partial center. For each $(x(\varepsilon), t(\varepsilon)) \in S\left(P_{\varepsilon}\right)$, we define $(\tau(\varepsilon), d(\varepsilon))$ by

$$
\tau(\varepsilon):=\varepsilon^{-1 / 2} t(\varepsilon) \text { and } d(\varepsilon):=\varepsilon^{-1 / 2}\left(x(\varepsilon)-x^{*}\right) .
$$

Our purpose in this section is to prove that $\left\{\left(\tau(\varepsilon), d^{0}(\varepsilon)\right)\right\}$ converges. We will consider the other components of $\{d(\varepsilon)\}$ in the last section.

The first result will concern $\left(\tau(\varepsilon), d_{I_{0}^{0}}^{0}(\varepsilon)\right)$ corresponding to the "principal part" in the cost function. Indeed, by Lemmas 3.2 and 3.3

$$
\frac{f_{\varepsilon}(x(\varepsilon), t(\varepsilon))}{\sqrt{\varepsilon}}=\frac{t}{\sqrt{\varepsilon}}+\sqrt{\varepsilon} \sum_{i \in I} \frac{\gamma_{i}+t(\varepsilon) \omega_{i}}{x_{i}^{0}(\varepsilon)}=\tau(\varepsilon)+\sum_{i \in I_{0}^{0}} \frac{\gamma_{i}}{d_{i}^{0}(\varepsilon)}+O(\sqrt{\varepsilon}) .
$$

Formula (4.5) suggests to consider the convex optimization problem

$$
\left\{\begin{array}{lll}
\underset{d, \tau}{\operatorname{Min}} & \tau+\sum_{i \in I_{0}^{0}} \frac{\gamma_{i}}{d_{i}^{0}}, & \\
\text { s.t. } & A d^{k}=0, & k=1, \ldots, K, \\
& \tau \omega_{i}-\sum_{1 \leq k \leq K} d_{i}^{k}=d_{i}^{0}, & i=1, \ldots, p, \\
& d_{I^{k}\left(x^{*}, 0\right)}^{k} \geq 0, & k=0, \ldots, K, \\
& d_{I_{0}^{0}}^{0}>0, \tau>0 . &
\end{array}\right.
$$

Note that for each $\varepsilon>0,(d(\varepsilon), \tau(\varepsilon))$ is feasible for $(U)$. Furthermore, the cost function of $(U)$ is strictly convex with respect to $d_{I_{0}^{0}}^{0}$. Hence, $d_{I_{0}^{0}}^{0}$ and consequently $\tau$ are constant over $S(U)$ whenever it is nonempty.

The next lemma describes two essential properties of $(U)$ that will be useful for proving the expansion concerning the principal part.

Lemma 4.1 The feasible set $F(U)$ is a convex cone and whenever $(\hat{d}, \hat{\tau}) \in S(U)$, we have

$$
\hat{\tau}=\sum_{i \in I_{0}^{0}} \frac{\gamma_{i}}{\hat{d}_{i}^{0}}
$$

Proof. The first property concerning $F(U)$ is obvious. Let $(\hat{d}, \hat{\tau}) \in S(U)$ and $\lambda>0$. Then $(\lambda \hat{d}, \lambda \hat{\tau})$ belongs to $F(U)$. Consider the auxiliary problem

$$
\operatorname{Min}_{\lambda>0} \lambda \hat{\tau}+\sum_{i \in I_{0}^{0}} \frac{\gamma_{i}}{\lambda \hat{d}_{i}^{0}}
$$

This problem is strictly convex and its optimal value is $v(U)$, while its unique solution is $\lambda=1$. The optimality condition for $\lambda=1$ achieves the proof.

The next lemma gives the expansions of $t(\varepsilon)$ and $x_{I_{0}^{0}}^{0}(\varepsilon)$ for $\varepsilon$ close to 0 . 
Lemma 4.2 The optimal set $S(U)$ is nonempty. Let $\left(d^{*}, \tau^{*}\right) \in S(U)$, then

$$
\begin{aligned}
& t(\varepsilon)=\tau^{*} \sqrt{\varepsilon}+o(\varepsilon), \\
& x_{I_{0}^{0}}^{0}(\varepsilon)=d_{I_{0}^{0}}^{* 0} \sqrt{\varepsilon}+O(\varepsilon) .
\end{aligned}
$$

Proof. By Lemma 3.2, $\tau(\varepsilon)$ is bounded. This implies, applying Lemma 3.1 to the linear constraints defining $F(L P)$ and those defining $S(L P)$, that $d_{I_{0}^{0}}^{0}(\varepsilon)$ is also bounded.

Let $\left(\tilde{\tau}, \tilde{d}_{I_{0}^{0}}^{0}\right)$ be a limit-point of $\left\{\left(\tau(\varepsilon), d_{I_{0}^{0}}^{0}(\varepsilon)\right)\right\}$. Applying again Lemma 3.1 to the constraints defining $F(U)$, there exists a vector $(\bar{d}, \bar{\tau}) \in F(U)$ such that $\left(\bar{\tau}, \bar{d}_{I_{0}^{0}}^{0}\right)=\left(\tilde{\tau}, \tilde{d}_{I_{0}^{0}}^{0}\right)$. For each $(d, \tau)$ in $F(U)$, define

$$
(\bar{x}(\varepsilon), \bar{t}(\varepsilon)):=\left(x^{*}, 0\right)+\sqrt{\varepsilon}(d, \tau) .
$$

As this vector belongs to $F\left(P_{\varepsilon}\right)$, we have

$$
t(\varepsilon)+\varepsilon \sum_{i \in I} \frac{\gamma_{i}+t(\varepsilon) \omega_{i}}{x_{i}^{0}(\varepsilon)} \leq \bar{t}(\varepsilon)+\varepsilon \sum_{i \in I} \frac{\gamma_{i}+\bar{t}(\varepsilon) \omega_{i}}{\bar{x}_{i}^{0}(\varepsilon)} .
$$

Dividing by $\sqrt{\varepsilon}$, we obtain

$$
\begin{aligned}
\tau(\varepsilon)- & +\sum_{i \in I_{0}^{0}}\left[\frac{\gamma_{i}}{d_{i}^{0}(\varepsilon)}-\frac{\gamma_{i}}{d_{i}^{0}}\right] \leq \\
& \sqrt{\varepsilon} \sum_{i \in I_{0}^{0}}\left[\frac{\tau \omega_{i}}{d_{i}^{0}}-\frac{\tau(\varepsilon) \omega_{i}}{d_{i}^{0}(\varepsilon)}\right]+\sqrt{\varepsilon} \sum_{i \notin I_{0}^{0}}\left[\frac{\gamma_{i}+\bar{t}(\varepsilon) \omega_{i}}{\left(x^{*}\right)_{i}^{0}+\sqrt{\varepsilon} d_{i}^{0}}-\frac{\gamma_{i}+t(\varepsilon) \omega_{i}}{\left(x^{*}\right)_{i}^{0}+\sqrt{\varepsilon} d_{i}^{0}(\varepsilon)}\right],
\end{aligned}
$$

and then

$$
\tau(\varepsilon)-\tau+\sum_{i \in I_{0}^{0}}\left[\frac{\gamma_{i}}{d_{i}^{0}(\varepsilon)}-\frac{\gamma_{i}}{d_{i}^{0}}\right] \leq \sqrt{\varepsilon} \sum_{i \in I_{0}^{0}}\left[\frac{\tau \omega_{i}}{d_{i}^{0}}-\frac{\tau(\varepsilon) \omega_{i}}{d_{i}^{0}(\varepsilon)}\right]+o(\sqrt{\varepsilon}) .
$$

Passing to the limit, we obtain that

$$
\bar{\tau}+\sum_{i \in I_{0}^{0}} \frac{\gamma_{i}}{\bar{d}_{i}^{0}} \leq \tau+\sum_{i \in I_{0}^{0}} \frac{\gamma_{i}}{d_{i}^{0}} .
$$

Therefore $(\bar{d}, \bar{\tau}) \in S(U)$ and then $S(U) \neq \emptyset$ and $\left(\bar{d}_{I_{0}^{0}}^{0}, \bar{\tau}\right)=\left(\left(d^{*}\right)_{I_{0}^{0}}^{0}, \tau^{*}\right)$.

i. For $\varepsilon>0$, using the definition of $S\left(P_{\varepsilon}\right)$, we have

$$
(d(\varepsilon), \tau(\varepsilon)) \in \underset{(d, \tau) \in F(U)}{\operatorname{argmin}} \tau+\sum_{i \in I_{0}^{0}} \frac{\gamma_{i}+\sqrt{\varepsilon} \tau \omega_{i}}{d_{i}^{0}}+\sqrt{\varepsilon} \sum_{i \notin I_{0}^{0}} \frac{\gamma_{i}+\sqrt{\varepsilon} \tau \omega_{i}}{\left(x^{*}\right)_{i}^{0}+\sqrt{\varepsilon} d_{i}^{0}} .
$$

$\mathrm{RR} \mathrm{n}^{\circ} 3133$ 
Since $\lambda(d(\varepsilon), \tau(\varepsilon)) \in F(U)$ for each $\lambda>0$, we have

$$
1 \in \underset{\lambda>0}{\operatorname{argmin}} \lambda \tau(\varepsilon)+\sum_{i \in I_{0}^{0}} \frac{\gamma_{i}+\sqrt{\varepsilon} \lambda \tau(\varepsilon) \omega_{i}}{\lambda d_{i}^{0}(\varepsilon)}+\sqrt{\varepsilon} \sum_{i \notin I_{0}^{0}} \frac{\gamma_{i}+\sqrt{\varepsilon} \lambda \tau(\varepsilon) \omega_{i}}{\left(x^{*}\right)_{i}^{0}+\sqrt{\varepsilon} \lambda d_{i}^{0}(\varepsilon)} .
$$

The first-order optimality condition implies after simplification that

$$
\tau(\varepsilon)-\sum_{i \in I_{0}^{0}} \frac{\gamma_{i}}{d_{i}^{0}(\varepsilon)}+\sqrt{\varepsilon} \sum_{i \notin I_{0}^{0}} \frac{\sqrt{\varepsilon} \tau(\varepsilon) \omega_{i}\left(x^{*}\right)_{i}^{0}-\sqrt{\varepsilon} \gamma_{i} d_{i}^{0}(\varepsilon)}{\left[\left(x^{*}\right)_{i}^{0}+\sqrt{\varepsilon} d_{i}^{0}(\varepsilon)\right]^{2}}=0 .
$$

As $\left(\sqrt{\varepsilon} d^{0}(\varepsilon), \sqrt{\varepsilon} \tau(\varepsilon)\right)$ converges to 0 by Lemma 3.2 and Lemma 3.3 , we get

$$
\tau(\varepsilon)=\sum_{i \in I_{0}^{0}} \frac{\gamma_{i}}{d_{i}^{0}(\varepsilon)}+o(\sqrt{\varepsilon}) .
$$

Since $(d(\varepsilon), \tau(\varepsilon)) \in F(U)$, applying (4.6) for $(d, \tau)=\left(d^{*}, \tau^{*}\right)$, we have

$$
0 \leq \tau(\varepsilon)-\tau^{*}+\sum_{i \in I_{0}^{0}}\left[\frac{\gamma_{i}}{d_{i}^{0}(\varepsilon)}-\frac{\gamma_{i}}{\left(d^{*}\right)_{i}^{0}}\right] \leq \sqrt{\varepsilon} \sum_{i \in I_{0}^{0}}\left[\frac{\tau^{*} \omega_{i}}{\left(d^{*}\right)_{i}^{0}}-\frac{\tau(\varepsilon) \omega_{i}}{d_{i}^{0}(\varepsilon)}\right]+o(\sqrt{\varepsilon}) .
$$

Then, the convergence of $\left(d_{I_{0}^{0}}^{0}(\varepsilon), \tau(\varepsilon)\right)$ to $\left(\left(d^{*}\right)_{I_{0}^{0}}^{0}, \tau^{*}\right)$ implies that

$$
0 \leq \tau(\varepsilon)-\tau^{*}+\sum_{i \in I_{0}^{0}}\left[\frac{\gamma_{i}}{d_{i}^{0}(\varepsilon)}-\frac{\gamma_{i}}{\left(d^{*}\right)_{i}^{0}}\right] \leq o(\sqrt{\varepsilon}) .
$$

By Lemma 4.1 and (4.8), we conclude that

$$
0 \leq 2 \tau(\varepsilon)-2 \tau^{*}+o(\sqrt{\varepsilon}) \leq o(\sqrt{\varepsilon}) .
$$

ii. We can write $S(U)$ as follows

$$
S(U)=\left\{(d, \tau) \in F(U), \quad d_{I_{0}^{0}}^{0}=\left(d^{*}\right)_{I_{0}^{0}}^{0} \quad \text { and } \quad \tau=\tau^{*}\right\} .
$$

We know that $(d(\varepsilon), \tau(\varepsilon)) \in F(U)$ and $\left(d_{I_{0}^{0}}^{0}(\varepsilon), \tau(\varepsilon)\right)$ converges to $\left(\left(d^{*}\right)_{I_{0}^{0}}^{0}, \tau^{*}\right)$.

By Lemma 3.1, for each $\varepsilon>0$ there exists $\left(\tilde{d}_{\varepsilon}, \tau^{*}\right) \in S(U)$ such that

$$
\left\|\tilde{d}_{\varepsilon}-d(\varepsilon)\right\| \leq \delta\left\|\left(\left(d^{*}\right)_{I_{0}^{0}}^{0}-d_{I_{0}^{0}}^{0}(\varepsilon), \tau^{*}-\tau(\varepsilon)\right)\right\|=o(1),
$$

where $\delta$ is a positive constant independent of $\varepsilon$.

Rewriting (4.6), for $(d, \tau)=\left(\tilde{d}_{\varepsilon}, \tau^{*}\right)$, we have

$$
\begin{aligned}
\tau(\varepsilon)-\tau^{*} & +\sum_{i \in I_{0}^{0}}\left[\frac{\gamma_{i}}{d_{i}^{0}(\varepsilon)}-\frac{\gamma_{i}}{\left(d^{*}\right)_{i}^{0}}\right] \leq \\
& \sqrt{\varepsilon}\left(\sum_{i \in I_{0}^{0}}\left[\frac{\tau^{*} \omega_{i}}{\left(d^{*}\right)_{i}^{0}}-\frac{\tau(\varepsilon) \omega_{i}}{d_{i}^{0}(\varepsilon)}\right]+\sum_{i \notin I_{0}^{0}}\left[\frac{\gamma_{i}+\sqrt{\varepsilon} \tau^{*} \omega_{i}}{\left(x^{*}\right)_{i}^{0}+\sqrt{\varepsilon}\left(\tilde{d}_{\varepsilon}\right)_{i}^{0}}-\frac{\gamma_{i}+\sqrt{\varepsilon} \tau(\varepsilon) \omega_{i}}{\left(x^{*}\right)_{i}^{0}+\sqrt{\varepsilon} d_{i}^{0}(\varepsilon)}\right]\right) .
\end{aligned}
$$

INRIA 
Since $\left(d(\varepsilon), \tau(\varepsilon) \in F(U)\right.$ and $\left(d^{*}, \tau^{*}\right) \in S(U)$ and since the cost function of (U) is strictly convex with respect to $d_{I_{0}^{0}}^{0}$, there exists $\beta>0$ such that

$$
\tau(\varepsilon)-\tau^{*}+\sum_{i \in I_{0}^{0}}\left[\frac{\gamma_{i}}{d_{i}^{0}(\varepsilon)}-\frac{\gamma_{i}}{\left(d^{*}\right)_{i}^{0}}\right] \geq \beta\left\|\left(d^{*}\right)_{I_{0}^{0}}^{0}-d_{I_{0}^{0}}^{0}(\varepsilon)\right\|^{2} .
$$

To obtain the order of magnitude of $d_{I_{0}^{0}}^{0}(\varepsilon)-\left(d^{*}\right)_{I_{0}^{0}}^{0}$, we will give estimate of the right handside of (4.10), depending only on $d_{I_{0}^{0}}^{0}(\varepsilon)-\left(d^{*}\right)_{I_{0}^{0}}^{0}$ and $\varepsilon$.

Using (i) and (4.9), for $i \notin I_{0}^{0}$, we have

$$
\begin{aligned}
\frac{\gamma_{i}+\sqrt{\varepsilon} \tau^{*} \omega_{i}}{\left(x^{*}\right)_{i}^{0}+\sqrt{\varepsilon}\left(\tilde{d}_{\varepsilon}\right)_{i}^{0}}-\frac{\gamma_{i}+\sqrt{\varepsilon} \tau(\varepsilon) \omega_{i}}{\left(x^{*}\right)_{i}^{0}+\sqrt{\varepsilon} d_{i}^{0}(\varepsilon)} & =\frac{\sqrt{\varepsilon} \tau^{*} \omega_{i}}{\left(x^{*}\right)_{i}^{0}+\sqrt{\varepsilon}\left(\tilde{d}_{\varepsilon}\right)_{i}^{0}}-\frac{\sqrt{\varepsilon} \tau(\varepsilon) \omega_{i}}{\left(x^{*}\right)_{i}^{0}+\sqrt{\varepsilon} d_{i}^{0}(\varepsilon)} \\
& +\frac{\gamma_{i} \sqrt{\varepsilon}\left(d_{i}^{0}(\varepsilon)-\left(\tilde{d}_{\varepsilon}\right)_{i}^{0}\right)}{\left.\left(\left(x^{*}\right)_{i}^{0}+\sqrt{\varepsilon}\left(\tilde{d}_{\varepsilon}\right)_{i}^{0}\right)\right)\left(\left(x^{*}\right)_{i}^{0}+\sqrt{\varepsilon} d_{i}^{0}(\varepsilon)\right)} \\
& =o(1) .
\end{aligned}
$$

Furthermore, using (ii) and since $d_{I_{0}^{0}}^{0}(\varepsilon)$ converges to $\left(d^{*}\right)_{I_{0}^{0}}^{0}>0$, we have

$$
\begin{aligned}
\frac{\tau^{*} \omega_{i}}{\left(d^{*}\right)_{i}^{0}}-\frac{\tau(\varepsilon) \omega_{i}}{d_{i}^{0}(\varepsilon)} & =\tau^{*} \omega_{i}\left[\frac{1}{\left(d^{*}\right)_{i}^{0}}-\frac{1}{d_{i}^{0}(\varepsilon)}\right]+\frac{o(\sqrt{\varepsilon})}{d_{i}^{0}(\varepsilon)} \\
& \leq \tau^{*} \omega_{i} \frac{\left\|\left(d^{*}\right)_{I_{0}^{0}}^{0}-d_{I_{0}^{0}}^{0}(\varepsilon)\right\|}{\left(d^{*}\right)_{i}^{0} d_{i}^{0}(\varepsilon)}+o(\sqrt{\varepsilon}) .
\end{aligned}
$$

Then the inequality (4.10) implies that

$$
\tau(\varepsilon)-\tau^{*}+\sum_{i \in I_{0}^{0}}\left[\frac{\gamma_{i}}{d_{i}^{0}(\varepsilon)}-\frac{\gamma_{i}}{\left(d^{*}\right)_{i}^{0}}\right] \leq O(\sqrt{\varepsilon})\left\|\left(d^{*}\right)_{I_{0}^{0}}^{0}-d_{I_{0}^{0}}^{0}(\varepsilon)\right\|+o(\varepsilon) .
$$

The proof is then complete using (4.13) and (4.11) $\square$

The second part of this section is devoted to the analysis of the other components of $\left\{d^{0}(\varepsilon)\right\}_{\varepsilon>0}$. In this study and also when doing the expansion of the cost function, we will meet the following two optimization problems.

$$
\begin{gathered}
\operatorname{Min}_{d}\left\{-\sum_{i \notin I_{0}^{0}} \frac{\gamma_{i} d_{i}^{0}}{\left(x_{i}^{* 0}\right)^{2}}, \quad\left(d, \tau^{*}\right) \in S(U)\right\}, \\
\operatorname{Min}_{d}\left\{2 \sum_{i \notin I_{0}^{0}} \frac{\gamma_{i}\left(d_{i}^{0}\right)^{2}}{\left(x_{i}^{* 0}\right)^{3}}-\sum_{i \notin I_{0}^{0}} \frac{\tau^{*} \omega_{i} d_{i}^{0}}{\left(x_{i}^{*}\right)^{2}}, \quad d \in S(T)\right\} .
\end{gathered}
$$

$\mathrm{RR} \mathrm{n}^{\circ} 3133$ 
By Lemma 4.2, $t(\varepsilon)=\sqrt{\varepsilon}+o(\varepsilon)$ and $x(\varepsilon)$ is a solution of

$$
\left\{\begin{array}{lll}
\operatorname{Min}_{x} & \sum_{i \notin I_{0}^{0}} \frac{\gamma_{i}+t(\varepsilon) \omega_{i}}{x_{i}^{0}}, & \\
\text { s.t. } & A x^{k}=b^{k}, & k=1, \ldots, K, \\
& \gamma_{i}+t(\varepsilon) \omega_{i}-\sum_{1 \leq k \leq K} x_{i}^{k}=x_{i}^{0}, & i=1, \ldots, p, \\
& x^{k} \geq 0, & k=0, \ldots, K, \\
& x_{I_{0}^{0}}^{0}=x_{I_{0}^{0}}^{0}(\varepsilon)=\sqrt{\varepsilon} d_{I_{0}^{0}}^{*}+O(\varepsilon) . &
\end{array}\right.
$$

Remark. The above problem can be interpreted as a perturbation of the limit problem

$$
\left\{\begin{array}{lll}
\underset{x}{\operatorname{Min}} & \sum_{i \notin I_{0}^{0}} \frac{\gamma_{i}}{x_{i}}, & \\
\text { s.t. } & A x^{k}=b^{k}, & k=1, \ldots, K, \\
& \gamma_{i}-\sum_{1 \leq k \leq K} x_{i}^{k}=x_{i}^{0}, & i=1, \ldots, p, \\
& x^{k} \geq 0, & k=0, \ldots, K, \\
& x_{I_{0}^{0}}^{0}=0 &
\end{array}\right.
$$

that satisfies $S(\tilde{P}) \times\{0\}=S(R)$.

Lemma 4.3 The sequence $\left\{d^{0}(\varepsilon)\right\}_{\varepsilon>0}$ is bounded.

Proof. We first prove that $S(T) \neq 0$. Indeed, this result will be necessary for the rest of the proof. Since $(T)$ is a feasible linear program, we only need to prove that $v(T)>-\infty$. Suppose the contrary, then there exists a feasible descent direction $y$ satisfying:

$$
\begin{array}{lrl}
-\sum_{i \notin I_{0}^{0}} \frac{y_{i}^{0}}{\left(x_{i}^{* 0}\right)^{2}}<0, & y_{I_{0}^{0}}^{0}=0, & y_{I^{k}\left(x^{*}, 0\right)}^{k} \geq 0, \quad k=0, \ldots, K, \\
A y^{k}=0, \quad k=1, \ldots, K, & -\sum_{1 \leq k \leq K} y_{i}^{k}=y_{i}^{0}, \quad i=1, \ldots, p .
\end{array}
$$

Then, for $\mu>0$ small enough, we have

$$
x^{\mu}:=x^{*}+\mu y \in F(\tilde{P}) \quad \text { and } \quad \sum_{i \notin I_{0}^{0}} \frac{\gamma_{i}}{x_{i}^{\mu 0}}<\sum_{i \notin I_{0}^{0}} \frac{\gamma_{i}}{x_{i}^{* 0}} .
$$

This contradicts the optimality of $x^{*}$ for $(\tilde{P})$.

In order to prove that $\left\{d^{0}(\varepsilon)\right\}$ is bounded, we will give two estimates of $v\left(\tilde{P}_{\varepsilon}\right)$ depending on $\varepsilon$ 
and $\left\|x \frac{0}{I_{0}^{0}}(\varepsilon)-\left(x^{*}\right) \frac{0}{I_{0}^{0}}\right\|$. These two estimates will then imply that $\left\|x \frac{0}{I_{0}^{0}}(\varepsilon)-\left(x^{*}\right) \frac{0}{I_{0}^{0}}\right\|=O(\sqrt{\varepsilon})$. Let $d \in S(T)$. Since $t(\varepsilon)=\sqrt{\varepsilon} \tau^{*}+o(\varepsilon)$, Lemma 3.1 applied to the set $F\left(\tilde{P}_{\varepsilon}\right)$ ensures that

$$
\operatorname{dist}\left(x^{*}+\sqrt{\varepsilon} d, F\left(\tilde{P}_{\varepsilon}\right)\right)=O(\varepsilon) .
$$

So there exists a vector $\tilde{x}(\varepsilon):=x^{*}+\sqrt{\varepsilon} d+O(\varepsilon)$ belonging to $F\left(\tilde{P}_{\varepsilon}\right)$.

Therefore, an upper bound of $v\left(\tilde{P}_{\varepsilon}\right)$ is

$$
\begin{aligned}
v\left(\tilde{P}_{\varepsilon}\right)=\sum_{i \notin I_{0}^{0}} \frac{\gamma_{i}+t(\varepsilon) \omega_{i}}{x_{i}^{0}(\varepsilon)} & \leq \sum_{i \notin I_{0}^{0}} \frac{\gamma_{i}+t(\varepsilon) \omega_{i}}{\left(x^{*}\right)_{i}^{0}+\sqrt{\varepsilon} d_{i}^{0}+O(\varepsilon)} \\
& \leq \sqrt{\varepsilon} v(T)+\sum_{i \notin I_{0}^{0}}\left(\frac{\gamma_{i}}{\left(x^{*}\right)_{i}^{0}}+\sqrt{\varepsilon} \frac{\tau^{*} \omega_{i}}{\left(x^{*}\right)_{i}^{0}}\right)+O(\varepsilon) .
\end{aligned}
$$

By Lemma 4.2 ,

$$
(d(\varepsilon), \tau(\varepsilon)) \in F(U), \quad \tau(\varepsilon)=\tau^{*}+o(\sqrt{\varepsilon}) \quad \text { and } \quad d_{I_{0}^{0}}^{0}(\varepsilon)=\left(d^{*}\right)_{I_{0}^{0}}^{0}+O(\sqrt{\varepsilon}) .
$$

Applying again Lemma 3.1, there exists $\left(\bar{d}(\varepsilon), \tau^{*}\right) \in S(U)$ such that

$$
\bar{d}(\varepsilon)-d(\varepsilon)=O(\sqrt{\varepsilon}) .
$$

For $i \notin I_{0}^{0}$ and $\varepsilon$ small enough, we have

$$
\frac{t(\varepsilon) \omega_{i}}{x_{i}^{0}(\varepsilon)}=\frac{\sqrt{\varepsilon} \tau^{*} \omega_{i}}{x_{i}^{0}(\varepsilon)}+o(\varepsilon),
$$

and

$$
\begin{aligned}
\frac{\gamma_{i}}{x_{i}^{0}(\varepsilon)} & =\frac{\gamma_{i}}{\left(x^{*}\right)_{i}^{0}}-\frac{\gamma_{i} \sqrt{\varepsilon} d_{i}^{0}(\varepsilon)}{\left(\left(x^{*}\right)_{i}^{0}\right)^{2}}+\frac{2 \gamma_{i}\left[x_{i}^{0}(\varepsilon)-\left(x^{*}\right)_{i}^{0}\right]^{2}}{\left(\left(x^{*}\right)_{i}^{0}\right)^{3}}+o\left(\left[x_{i}^{0}(\varepsilon)-\left(x^{*}\right)_{i}^{0}\right]^{2}\right), \\
& \geq \frac{\gamma_{i}}{\left(x^{*}\right)_{i}^{0}}-\frac{\gamma_{i} \sqrt{\varepsilon} d_{i}^{0}(\varepsilon)}{\left(\left(x^{*}\right)_{i}^{0}\right)^{2}}+\frac{\gamma_{i}\left[x_{i}^{0}(\varepsilon)-\left(x^{*}\right)_{i}^{0}\right]^{2}}{\left(\left(x^{*}\right)_{i}^{0}\right)^{3}}
\end{aligned}
$$

Then,

$$
v\left(\tilde{P}_{\varepsilon}\right) \geq \sum_{i \notin I_{0}^{0}}\left(\frac{\gamma_{i}}{\left(x^{*}\right)_{i}^{0}}+\sqrt{\varepsilon}\left[\frac{-\gamma_{i} d_{i}^{0}(\varepsilon)}{\left(\left(x^{*}\right)_{i}^{0}\right)^{2}}+\frac{\tau^{*} \omega_{i}}{x_{i}^{0}(\varepsilon)}\right]+\frac{\gamma_{i}\left[x_{i}^{0}(\varepsilon)-\left(x^{*}\right)_{i}^{0}\right]^{2}}{\left(\left(x^{*}\right)_{i}^{0}\right)^{3}}\right)+o(\varepsilon),
$$

and by $(4.15)$, a lower bound for $v\left(\tilde{P}_{\varepsilon}\right)$ is

$$
v\left(\tilde{P}_{\varepsilon}\right) \geq \sqrt{\varepsilon} v(T)+\sum_{i \notin I_{0}^{0}}\left(\frac{\gamma_{i}}{\left(x^{*}\right)_{i}^{0}}+\frac{\gamma_{i}\left[x_{i}^{0}(\varepsilon)-\left(x^{*}\right)_{i}^{0}\right]^{2}}{\left(\left(x^{*}\right)_{i}^{0}\right)^{3}}+\sqrt{\varepsilon} \frac{\tau^{*} \omega_{i}}{x_{i}^{0}(\varepsilon)}\right)+O(\varepsilon) .
$$


Thus (4.14) and (4.16) yield to

$$
\sum_{i \notin I_{0}^{0}} \frac{\gamma_{i}\left(x_{i}^{0}(\varepsilon)-\left(x^{*}\right)_{i}^{0}\right)^{2}}{\left(\left(x^{*}\right)_{i}^{0}\right)^{3}} \leq \sqrt{\varepsilon} \sum_{i \notin I_{0}^{0}}\left(\frac{\tau^{*} \omega_{i}}{\left(x^{*}\right)_{i}^{0}}-\frac{\tau^{*} \omega_{i}}{x_{i}^{0}(\varepsilon)}\right)+O(\varepsilon) .
$$

For $i \notin I_{0}^{0},\left(x^{*}\right)_{i}^{0}>0$ and then there exist two constants $\alpha>0$ and $\beta \geq 0$ independent of $\varepsilon$ such that

$$
\alpha\left\|x \frac{0}{I_{0}^{0}}(\varepsilon)-\left(x^{*}\right) \frac{0}{I_{0}^{0}}\right\|^{2} \leq \beta \sqrt{\varepsilon}\left\|x \frac{0}{\bar{I}_{0}^{0}}(\varepsilon)-\left(x^{*}\right) \frac{0}{I_{0}^{0}}\right\|+O(\varepsilon),
$$

completing the proof. $\square$

Lemma 4.4 The sequence $\left\{d^{0}(\varepsilon)\right\}_{\varepsilon>0}$ is convergent.

Proof. By Lemma 4.3, $d^{0}(\varepsilon)$ has at least a limit point. Let $\bar{d} \in F(U)$ be such that $\bar{d}^{0}$ is a limit-point of $d^{0}(\varepsilon)$. Such a vector $\bar{d}$ exists by applying Lemma 3.1 to $F(U)$. By Lemma $4.2,\left(\bar{d}, \tau^{*}\right) \in S(U)$. If $S(U)$ is reduced to a singleton the conclusion follows. Else, let $\left(\tilde{d}, \tau^{*}\right) \in S(U)$ be such that $\tilde{d} \neq \bar{d}$. Consider a sequence $\left(d^{0}\left(\varepsilon_{k}\right), \tau\left(\varepsilon_{k}\right)\right)$ converging to $\left(\bar{d}^{0}, \tau^{*}\right)$. Set

$$
\left(\tilde{d}\left(\varepsilon_{k}\right), \tilde{\tau}\left(\varepsilon_{k}\right)\right)=\left(\tilde{d}, \tau^{*}\right)-\left(\bar{d}, \tau^{*}\right)+\left(d\left(\varepsilon_{k}\right), \tau\left(\varepsilon_{k}\right)\right) .
$$

We have $\tilde{d}_{I_{0}^{0}}^{0}\left(\varepsilon_{k}\right)=d_{I_{0}^{0}}^{0}\left(\varepsilon_{k}\right), \tilde{\tau}\left(\varepsilon_{k}\right)=\tau\left(\varepsilon_{k}\right)$ and $\lim _{\varepsilon_{k} \downarrow 0}\left(\tilde{d}\left(\varepsilon_{k}\right), \tilde{\tau}\left(\varepsilon_{k}\right)\right)=\left(\tilde{d}, \tau^{*}\right)$.

Choosing $(d, \tau)=\left(\tilde{d}\left(\varepsilon_{k}\right), \tilde{\tau}\left(\varepsilon_{k}\right)\right)$ in (4.6), we have

$$
\sum_{i \notin I_{0}^{0}} \frac{\gamma_{i}+t\left(\varepsilon_{k}\right) \omega_{i}}{x_{i}^{* 0}+\sqrt{\varepsilon_{k}} d_{i}^{0}\left(\varepsilon_{k}\right)} \leq \sum_{i \notin I_{0}^{0}} \frac{\gamma_{i}+t\left(\varepsilon_{k}\right) \omega_{i}}{x_{i}^{* 0}+\sqrt{\varepsilon_{k}} \tilde{d}_{i}^{0}\left(\varepsilon_{k}\right)}
$$

and then

$$
\begin{aligned}
\sum_{i \notin I_{0}^{0}}\left[-\frac{\gamma_{i} \sqrt{\varepsilon_{k}} d_{i}^{0}\left(\varepsilon_{k}\right)}{\left(x^{* 0}\right)^{2}}+\frac{2 \gamma_{i} \varepsilon_{k}\left(d_{i}^{0}\left(\varepsilon_{k}\right)\right)^{2}}{\left(x^{* 0}{ }_{i}^{3}\right)^{3}}+\frac{\sqrt{\varepsilon_{k}} t\left(\varepsilon_{k}\right) \omega_{i}\left[\tilde{d}_{i}^{0}-\bar{d}_{i}^{0}\right]}{\left(x_{i}^{* 0}\right)^{2}}\right] \leq \\
\sum_{i \notin I_{0}^{0}} \gamma_{i}\left[-\frac{\sqrt{\varepsilon_{k}} \tilde{d}_{i}^{0}\left(\varepsilon_{k}\right)}{\left(x^{* 0}{ }_{i}\right)^{2}}+\frac{2 \varepsilon_{k}\left(\tilde{d}_{i}^{0}\left(\varepsilon_{k}\right)\right)^{2}}{\left(x^{* 0}{ }_{i}\right)^{3}}\right]+o\left(\varepsilon_{k}\right) .
\end{aligned}
$$

We obtain after a simple calculus

$$
\sum_{i \notin I_{0}^{0}}\left[2 \gamma_{i} \frac{\left(d_{i}^{0}\left(\varepsilon_{k}\right)\right)^{2}-\left(\tilde{d}_{i}^{0}\left(\varepsilon_{k}\right)\right)^{2}}{\left(x_{i}^{* 0}\right)^{3}}+\frac{\tau^{*} \omega_{i}\left[\tilde{d}_{i}^{0}-\bar{d}_{i}^{0}\right]}{\left(x_{i}^{* 0}\right)^{2}}\right] \leq \sum_{i \notin I_{0}^{0}} \frac{\gamma_{i}\left(\bar{d}_{i}^{0}-\tilde{d}_{i}^{0}\right)}{\sqrt{\varepsilon_{k}\left(x_{i}^{* 0}\right)^{2}}}+o(1) .
$$

Since the left hand-side is bounded and $\lim _{k \rightarrow+\infty} \frac{1}{\sqrt{\varepsilon}_{k}}=+\infty$, it follows that

$$
\sum_{i \notin I_{0}^{0}} \frac{\gamma_{i}\left[\bar{d}_{i}-\tilde{d}_{i}\right]}{\left(x_{i}^{* 0}\right)^{2}} \geq 0, \quad \text { for all } \quad\left(\tilde{d}, \tau^{*}\right) \in S(U)
$$

INRIA 
This inequality proves that $\bar{d}$ belongs to $S(T)$. Choosing $\tilde{d} \in S(T)$ in (4.20) and passing to the limit, we prove that $\bar{d}$ is also a solution of $T_{1}$. The proof is then complete since $d^{0}$ is constant over $S\left(T_{1}\right)$. $\mathrm{\square}$

Finally we can state a theorem that summarizes all results of this section:

Theorem 4.1 Every path $\{(x(\varepsilon), t(\varepsilon))\}$ such that $(x(\varepsilon), t(\varepsilon)) \in S\left(P_{\varepsilon}\right)$ satisfies

$$
\begin{aligned}
& t(\varepsilon)=\tau^{*} \sqrt{\varepsilon}+o(\varepsilon), \\
& x_{I_{0}^{0}}^{0}(\varepsilon)=\sqrt{\varepsilon}\left(d^{*}\right)_{I_{0}^{0}}^{0}+O(\varepsilon), \\
& x_{\bar{I}_{0}^{0}}^{0}(\varepsilon)=\left(x^{*}\right)_{\bar{I}_{0}^{0}}^{0}+\sqrt{\varepsilon}\left(d^{*}\right)_{\bar{I}_{0}^{0}}^{0}+o(\sqrt{\varepsilon}),
\end{aligned}
$$

where $\left(x^{*}, 0\right) \in S(R),\left(d^{*}, \tau^{*}\right) \in S(U)$ and $d^{*} \in S\left(T_{1}\right)$.

\section{Asymptotic expansion of the cost function}

In this section we study the cost function $v(\cdot): \varepsilon \rightarrow v\left(P_{\varepsilon}\right)$ and give an asymptotic expansion for $\varepsilon$ close to 0 . The method that we follow is reminiscent of asymptotic techniques [13] based on epiconvergence theory [1]. Here is the result of this section.

Theorem 5.1 The function $v(\cdot)$ satisfies

$$
v\left(P_{\varepsilon}\right)=\sqrt{\varepsilon} v(U)+\varepsilon\left(v(R)+\sum_{i \in I_{0}^{0}} \frac{\tau^{*} \omega_{i}}{\left(d^{*}\right)_{i}^{0}}\right)+o(\varepsilon),
$$

where $\left(\tau^{*}, d^{*}\right) \in S(U)$.

Proof. Let $\alpha$ be a nonnegative real number that we will choose next. Set

$$
\tilde{f}_{\varepsilon}(x, t):= \begin{cases}\frac{f_{\varepsilon}(x, t)-\alpha \sqrt{\varepsilon}}{\varepsilon} & \text { if }(x, t) \in F(L P) \\ +\infty & \text { else. }\end{cases}
$$

This rescaling is motivated by the expansion of the solutions. Indeed, the obtained expansion suggests that a part of the cost function is of order $\sqrt{\varepsilon}$. More about similar rescaling techniques in optimization problems can be found in [13].

Observe first that solving $\left(P_{\varepsilon}\right)$ is equivalent to solve the scaled problem $\operatorname{Min}_{x, t} \tilde{f}_{\varepsilon}(x, t)$.

Let $\left\{\left(x\left(\varepsilon_{k}\right), t\left(\varepsilon_{k}\right)\right)\right\}\left(\left(x\left(\varepsilon_{k}\right), t\left(\varepsilon_{k}\right)\right) \in S\left(P_{\varepsilon_{k}}\right)\right)$ be a sequence converging to a partial center $(\tilde{x}, 0)$ and consider an other sequence $\left\{\left(\bar{x}_{\varepsilon_{k}}, \bar{t}_{\varepsilon_{k}}\right)\right\}$ converging to $(\tilde{x}, 0)$ and defined by

$$
\bar{x}_{\varepsilon_{k}}=\tilde{x}+\sqrt{\varepsilon_{k}} d^{*} \quad \text { and } \quad \bar{t}_{\varepsilon_{k}}=\tau^{*} \sqrt{\varepsilon_{k}},
$$


where $\left(\tau^{*}, d^{*}\right)$ is a fixed solution of $(U)$. For each $\varepsilon_{k}$, we know that

$$
\tilde{f}_{\varepsilon_{k}}\left(x\left(\varepsilon_{k}\right), t\left(\varepsilon_{k}\right)\right) \leq \tilde{f}_{\varepsilon_{k}}\left(\bar{x}_{\varepsilon_{k}}, \bar{t}_{\varepsilon_{k}}\right) .
$$

Let us study the two hand-sides of this inequality. We have first

$$
\begin{aligned}
\tilde{f}_{\varepsilon_{k}}\left(x\left(\varepsilon_{k}\right), t\left(\varepsilon_{k}\right)\right) & =\frac{t\left(\varepsilon_{k}\right)}{\varepsilon_{k}}-\frac{\alpha}{\sqrt{\varepsilon_{k}}}+\sum_{i \in I_{0}^{0}}\left(\frac{\gamma_{i}}{x_{i}^{0}\left(\varepsilon_{k}\right)}+\frac{t\left(\varepsilon_{k}\right) \omega_{i}}{x_{i}^{0}\left(\varepsilon_{k}\right)}\right)+\sum_{i \notin I_{0}^{0}} \frac{\gamma_{i}+t\left(\varepsilon_{k}\right) \omega_{i}}{x_{i}^{0}\left(\varepsilon_{k}\right)}, \\
& =\frac{1}{\sqrt{\varepsilon_{k}}}\left[\tau\left(\varepsilon_{k}\right)+\sum_{i \in I_{0}^{0}} \frac{\gamma_{i}}{d_{i}^{0}\left(\varepsilon_{k}\right)}-\alpha\right]+\sum_{i \in I_{0}^{0}} \frac{\tau\left(\varepsilon_{k}\right) \omega_{i}}{d_{i}^{0}\left(\varepsilon_{k}\right)}+\sum_{i \notin I_{0}^{0}} \frac{\gamma_{i}+t\left(\varepsilon_{k}\right) \omega_{i}}{x_{i}^{0}\left(\varepsilon_{k}\right)} .
\end{aligned}
$$

(The notations $\tau\left(\varepsilon_{k}\right)$ and $d\left(\varepsilon_{k}\right)$ are those used in Section 3.)

Since $\left(d\left(\varepsilon_{k}\right), \tau\left(\varepsilon_{k}\right)\right) \in F(U)$, choosing $\alpha=v(U)$ we have

$$
\tilde{f}_{\varepsilon_{k}}\left(x\left(\varepsilon_{k}\right), t\left(\varepsilon_{k}\right)\right) \geq \sum_{i \in I_{0}^{0}} \frac{\tau\left(\varepsilon_{k}\right) \omega_{i}}{d_{i}^{0}\left(\varepsilon_{k}\right)}+\sum_{i \notin I_{0}^{0}} \frac{\gamma_{i}+t\left(\varepsilon_{k}\right) \omega_{i}}{x_{i}^{0}\left(\varepsilon_{k}\right)} .
$$

Passing to the limit and using Lemma 4.2, we obtain

$$
\liminf _{\varepsilon_{k} \downarrow 0} \tilde{f}_{\varepsilon_{k}}\left(x\left(\varepsilon_{k}\right), t\left(\varepsilon_{k}\right)\right) \geq \sum_{i \in I_{0}^{0}} \frac{\tau^{*} \omega_{i}}{\left(d^{*}\right)_{i}^{0}}+\sum_{i \notin I_{0}^{0}} \frac{\gamma_{i}}{\tilde{x}_{i}^{0}}=\sum_{i \in I_{0}^{0}} \frac{\tau^{*} \omega_{i}}{\left(d^{*}\right)_{i}^{0}}+v(R) .
$$

Now, consider the right hand-side of (5.22); we have

$$
\begin{aligned}
\tilde{f}_{\varepsilon_{k}}\left(\bar{x}_{\varepsilon_{k}}, \bar{t}_{\varepsilon_{k}}\right) & =\frac{\bar{t}_{\varepsilon_{k}}}{\varepsilon_{k}}-\frac{v(U)}{\sqrt{\varepsilon_{k}}}+\sum_{i \in I_{0}^{0}} \frac{\gamma_{i}}{\left(\bar{x}_{\varepsilon_{k}}\right)_{i}^{0}}+\sum_{i \in I_{0}^{0}} \frac{\bar{t}_{\varepsilon_{k}} \omega_{i}}{\left(\bar{x}_{\varepsilon_{k}}\right)_{i}^{0}}+\sum_{i \notin I_{0}^{0}} \frac{\gamma_{i}+\bar{t}_{\varepsilon_{k}} \omega_{i}}{\left(\bar{x}_{\varepsilon_{k}}\right)_{i}^{0}}, \\
& =\frac{1}{\sqrt{\varepsilon_{k}}}\left[\tau^{*}+\sum_{i \in I_{0}^{0}} \frac{\gamma_{i}}{\left(d^{*}\right)_{i}^{0}}-v(U)\right]+\sum_{i \in I_{0}^{0}} \frac{\tau^{*} \omega_{i}}{\left(d^{*}\right)_{i}^{0}}+\sum_{i \notin I_{0}^{0}} \frac{\gamma_{i}+\bar{t}_{\varepsilon_{k}} \omega_{i}}{\left(\bar{x}_{\varepsilon_{k}}\right)_{i}^{0}} .
\end{aligned}
$$

Since $\left(\tau^{*}, d^{*}\right) \in S(U)$, the last equality becomes

$$
\tilde{f}_{\varepsilon_{k}}\left(\bar{x}_{\varepsilon_{k}}, \bar{t}_{\varepsilon_{k}}\right)=\sum_{i \in I_{0}^{0}} \frac{\tau^{*} \omega_{i}}{\left(d^{*}\right)_{i}^{0}}+\sum_{i \notin I_{0}^{0}} \frac{\gamma_{i}+\bar{t}_{\varepsilon_{k}} \omega_{i}}{\left(\bar{x}_{\varepsilon_{k}}\right)_{i}^{0}}=\sum_{i \in I_{0}^{0}} \frac{\tau^{*} \omega_{i}}{\left(d^{*}\right)_{i}^{0}}+v(R)+o(1) .
$$

Passing to the limit and using (5.22), we have

$$
\limsup _{\varepsilon_{k} \downarrow 0} \tilde{f}_{\varepsilon_{k}}\left(x\left(\varepsilon_{k}\right), t\left(\varepsilon_{k}\right)\right) \leq \sum_{i \in I_{0}^{0}} \frac{\tau^{*} \omega_{i}}{\left(d^{*}\right)_{i}^{0}}+v(R) .
$$

Formulas (5.24) and (5.25) imply the result. $\square$

INRIA 


\section{Extensions}

In this section we consider a special path $\left\{(\tilde{x}(\varepsilon), \tilde{t}(\varepsilon)) \in S\left(P_{\varepsilon}\right)\right\}$ converging to some partial center $(\tilde{x}, 0)$. For this special path we establish an asymptotic expansion near 0 of all components of the solutions and not only $x^{0}$.

Let $(\tilde{x}(\varepsilon), \tilde{t}(\varepsilon))$ be defined by

$$
\tilde{t}(\varepsilon):=\underset{(x, t) \in S\left(P_{\varepsilon}\right)}{\operatorname{argmin}} t
$$

and

$$
\tilde{x}(\varepsilon):=\underset{(x, \tilde{t}(\varepsilon)) \in S\left(P_{\varepsilon}\right)}{\operatorname{argmin}} g(x):=\sum_{1 \leq k \leq K} \sum_{i \in I}\left(x_{i}^{k}-\tilde{x}_{i}^{k}\right)^{2} .
$$

Lemma 6.1 $\{(\tilde{x}(\varepsilon), \tilde{t}(\varepsilon))\}$ is well defined and $\lim _{\varepsilon \downarrow 0} \tilde{x}(\varepsilon)=\tilde{x}$.

Proof. Since $S\left(P_{\varepsilon}\right)$ is closed and bounded $\tilde{t}(\varepsilon)$ exists and is unique. Then, the strict convexity of $f_{\varepsilon}$ (resp. $g$ ) with respect to the components in $I^{0}$ (resp. in $I^{k}, 1 \leq k \leq$ $K)$ implies the uniquness of $\tilde{x}(\varepsilon)$. Furthermore, it is already proved in lemma 3.3 that $\{(\tilde{x}(\varepsilon), \tilde{t}(\varepsilon)\}$ is bounded and all its limit-points belong to $S(R)$.

Let $(\bar{x}, 0)=\lim _{k \rightarrow+\infty}\left(\tilde{x}\left(\varepsilon_{k}\right), \tilde{t}\left(\varepsilon_{k}\right)\right)$ for some sequence $\left\{\varepsilon_{k}\right\}$ converging to 0 .

Then

$$
(\tilde{x}, 0)=\lim _{k \rightarrow+\infty}\left(x\left(\varepsilon_{k}\right), t\left(\varepsilon_{k}\right)\right) \text { where }\left(x\left(\varepsilon_{k}\right), t\left(\varepsilon_{k}\right)\right)=\left(\tilde{x}\left(\varepsilon_{k}\right), \tilde{t}\left(\varepsilon_{k}\right)\right)+(\tilde{x}, 0)-(\bar{x}, 0) .
$$

Since $\bar{x}^{0}=\tilde{x}^{0}$, we have

$$
t\left(\varepsilon_{k}\right)=\tilde{t}\left(\varepsilon_{k}\right) \quad \text { and } \quad \bar{x}^{0}\left(\varepsilon_{k}\right)=\tilde{x}^{0}\left(\varepsilon_{k}\right),
$$

then (6.27) gives

$$
\sum_{1 \leq k^{\prime} \leq K} \sum_{i \in I}\left(\tilde{x}_{i}^{k^{\prime}}\left(\varepsilon_{k}\right)-\tilde{x}_{i}^{k^{\prime}}\right)^{2} \leq \sum_{1 \leq k^{\prime} \leq K} \sum_{i \in I}\left(x_{i}^{k^{\prime}}\left(\varepsilon_{k}\right)-\tilde{x}_{i}^{k^{\prime}}\right)^{2} .
$$

Passing to the limit we obtain that $\bar{x}=\tilde{x}$. $\square$

Consider like in the previous section $d(\varepsilon):=\varepsilon^{-1 / 2}(\tilde{x}(\varepsilon)-\tilde{x})$ and $\tau(\varepsilon):=\varepsilon^{-1 / 2} \tilde{t}(\varepsilon)$. From the results of Theorem 4.1, $\tilde{t}(\varepsilon)=\sqrt{\varepsilon} \tau^{*}+o(\varepsilon)$ and $\tilde{x}(\varepsilon)$ is a solution of the perturbed problem

$\left(\widetilde{P S}_{\varepsilon}\right) \quad\left\{\begin{array}{lll}\underset{x}{\operatorname{Min}} \quad \sum_{1 \leq k \leq K} \sum_{i \in I}\left(x_{i}^{k}-\tilde{x}_{i}^{k}\right)^{2}, & \\ \text { s.t. } & A x^{k}=b^{k}, & \\ & \gamma_{i}+\tilde{t}(\varepsilon) \omega_{i}-\sum_{1 \leq k \leq K} x_{i}^{k}=x_{i}^{0}, & i=1, \ldots, k, \\ & x \geq 0, \quad x^{0}=\tilde{x}^{0}+d^{* 0} \sqrt{\varepsilon}+o(\sqrt{\varepsilon}) .\end{array}\right.$ 
The corresponding limit problem is

$$
\left\{\begin{array}{cll}
\underset{x}{\operatorname{Min}} & \sum_{1 \leq k \leq K} \sum_{i \in I}\left(x_{i}^{k}-\tilde{x}_{i}^{k}\right)^{2}, & \\
\text { s.t. } & A x^{k}=b^{k}, & k=1, \ldots, K, \\
& \gamma_{i}-\sum_{1 \leq k \leq K} x_{i}^{k}=x_{i}^{0}, & i=1, \ldots, p, \\
& x \geq 0, \quad x^{0}=\tilde{x}^{0} . &
\end{array}\right.
$$

Remark. $F(\widetilde{P S}) \times\{0\}=S(R)$ and $S(\widetilde{P S})=\{\tilde{x}\}$.

Using Lemma 3.1, there exists $\hat{x}(\varepsilon) \in F\left(\widetilde{P S}_{\varepsilon}\right)$ such that

$$
\operatorname{dist}(\hat{x}(\varepsilon), \tilde{x})=O(\sqrt{\varepsilon})
$$

and in particular

$$
\sum_{1 \leq k \leq K} \sum_{i \in I}\left(\hat{x}_{i}^{k}(\varepsilon)-\tilde{x}_{i}^{k}\right)^{2}=O(\varepsilon)
$$

This obviously implies that

$$
v\left(\widetilde{P S}_{\varepsilon}\right)=\sum_{1 \leq k \leq K} \sum_{i \in I}\left(\tilde{x}_{i}^{k}(\varepsilon)-\tilde{x}_{i}^{k}\right)^{2}=O(\varepsilon),
$$

and consequently

$$
\operatorname{dist}(\tilde{x}(\varepsilon), \tilde{x})=O(\sqrt{\varepsilon}),
$$

or equivalently, the sequence $\{d(\varepsilon)\}_{\varepsilon>0}$ is bounded.

Lemma 6.2 $\tilde{x}(\varepsilon)=\tilde{x}+d^{*} \sqrt{\varepsilon}+o(\sqrt{\varepsilon})$, with

$$
\left\{d^{*}\right\}=\underset{d \in S\left(T_{1}\right)}{\operatorname{argmin}} \sum_{1 \leq k \leq K} \sum_{i \in I}\left(d_{i}^{k}\right)^{2} .
$$

Proof. Applying Theorem 4.1, $\lim _{\varepsilon \downarrow 0} \tau(\varepsilon)=\tau^{*}$ and any limit-point of $\{d(\varepsilon)\}$ belongs to $S\left(T_{1}\right)$. Let $\tilde{d}$ be one these limit-points and $\bar{d}$ be an other element of $S\left(T_{1}\right)$. We can write, for some sequence $\left\{\varepsilon_{k}\right\}$ converging to 0 , that

$$
\tilde{d}=\lim _{k \rightarrow+\infty} \tilde{d}\left(\varepsilon_{k}\right) \quad \text { and } \quad \bar{d}=\lim _{k \rightarrow+\infty} d\left(\varepsilon_{k}\right) \quad \text { with } \quad d\left(\varepsilon_{k}\right)=\tilde{d}\left(\varepsilon_{k}\right)-\tilde{d}+\bar{d} .
$$

Rewriting the definition of $\tilde{x}\left(\varepsilon_{k}\right)$ we obtain, since $\tilde{d}^{0}\left(\varepsilon_{k}\right)=\bar{d}^{0}\left(\varepsilon_{k}\right)$, that

$$
\sum_{1 \leq k \leq K} \sum_{i \in I}\left(\tilde{d}_{i}^{k}\left(\varepsilon_{k}\right)\right)^{2} \leq \sum_{1 \leq k \leq K} \sum_{i \in I}\left(\bar{d}_{i}^{k}\left(\varepsilon_{k}\right)\right)^{2}
$$

Passing to the limit the result holds.

INRIA 


\section{References}

[1] H. Atтouch, Variational convergences for functions and operators, Pitman Advanced Publishing Program (1984).

[2] A. Auslender, R. Cominetti And M. Haddou, Asymptotic analysis for penalty and barrier methods in convex and linear programming. Mathematics of Operations Research, to appear.

[3] J.F. Bonnans And A. Ioffe, Quadratic growth and stability in convex programming problems with multiple solutions. Journal of Convex Analysis 2(1995), pp. 41-57.

[4] J.F. Bonnans And A. Shapiro, Optimization problems with perturbations, a guided tour. Rapport de Recherche INRIA 2872 (1996). Submitted to the SIAM Review.

[5] C.W. CARRolL, The created response surface technique for optimizing nonlinear restrained systems. Operations Research 9(1961), pp. 169-184.

[6] A.V. Fiacco (ed.), Mathematical programming with perturbations. Marcel Dekker, to appear.

[7] D. Den Hertog, C. Roos and T. Terlaky, Inverse barrier methods for linear programming Report No 91-27, Faculty of Mathematics and Informatics, Delft University of Technology, Delft, Holland.

[8] C.C. Gonzaga, Path following methods for linear programming, SIAM Review 34(1992), pp. 167-227.

[9] A. Hoffmann, On approximate solutions of systems of inequalities. J.Res. National Bureau Standards, Sect. B 49 (1952), pp. 263-265.

[10] L. Kleinrock, Communications, Nets, Stochastic message flow and delay. Dover (1972).

[11] Y. Nesterov And A. Nemirovski, Interior-point polynomial algorithms in convex programming. SIAM Publications, SIAM, Philadelphia, 1994.

[12] A. Shapiro, Perturbation theory of nonlinear programs when the set of optimal solutions is not a singleton, Applied Mathematics \& Optimization 18 (1988), pp. 215-229.

[13] D. Torralba, Développement asymptotiques pour les méthodes d'approximation par viscosité. C.R. Acad. Sci. Paris, t. 322, Série I (1996), pp. 123-128.

$\mathrm{RR} \mathrm{n}^{\circ} 3133$ 
Unité de recherche INRIA Lorraine, Technopôle de Nancy-Brabois, Campus scientifique, 615 rue du Jardin Botanique, BP 101, 54600 VILLERS LÈS NANCY

Unité de recherche INRIA Rennes, Irisa, Campus universitaire de Beaulieu, 35042 RENNES Cedex

Unité de recherche INRIA Rhône-Alpes, 655, avenue de l'Europe, 38330 MONTBONNOT ST MARTIN

Unité de recherche INRIA Rocquencourt, Domaine de Voluceau, Rocquencourt, BP 105, 78153 LE CHESNAY Cedex

Unité de recherche INRIA Sophia-Antipolis, 2004 route des Lucioles, BP 93, 06902 SOPHIA-ANTIPOLIS Cedex

Éditeur
INRIA, Domaine de Voluceau, Rocquencourt, BP 105, 78153 LE CHESNAY Cedex (France)

ISSN 0249-6399 